\title{
THE METRIC DOCUMENTATION OF CHAM TOWERS IN VIETNAM BY SPHERICAL PHOTOGRAMMETRY
}

\author{
G. Fangi, E. S. Malinverni, A. N. Tassetti
}

Università Politecnica Marche, Ancona, Italy

g.fangi;e.s.malinverni;n.tassetti@univpm.it

KEY WORDS: Spherical photogrammetry, surveying, architecture, archaeology, documentation

\begin{abstract}
:
The Università Politecnica delle Marche is charged of the study, survey and documentation of the Chan towers in Vietnam. The towers are spread near the coastline from latitude $16^{\circ} 30^{\prime}$, to latitude of $10^{\circ} 56^{\prime}$. The Champa civilization flourished from $8^{\text {th }}$ century till $17^{\text {th }}$ century produced the Cham towers, places of religious worship. The ancient Cham people created a developed culture expressed in the architecture in the towers, which were Buddhist temples. Roughly there are six different architectonic styles and two different layouts in plan. One lay-out is that one main tower is surrounded by smaller service buildings, like walls, doors, gates; the other layout is a set of three aligned towers. We carried out the documentation with three different techniques, laser scanning, dense point clouds generation with SfM (Structure from Motion) algorithm, and mainly by Panoramic Spherical Photogrammetry (PSP). Laser scanning is indeed the best instrument but sometimes its availability is very limited for different practical and logistic reasons. The point cloud generation produced good results, but it has some problems and limitations of management too. On the other hand PSP is very quick technique in the taking phase, but the restitution is rather slow. PSP is suitable for a good metric documentation, making the Cham tower project the main project of documentation executed with spherical photogrammetry.
\end{abstract}

\section{INTRODUCTION}

The Università Politecnica delle Marche, Ancona, Italy, is carrying out a research on the Cham towers in Vietnam. The kingdom of Champa existed from the $8^{\text {th }}$ to the $17^{\text {th }}$ century A.D. in Vietnam and it was located near the coast of the southern part of the Vietnam. The ancient Cham people created a developed culture expressed in the architecture in the so-called towers, which were baddish temples. The Cham towers are classified with different styles: Antique style (from $8^{\text {th }}$ to $9^{\text {th }} \mathrm{c}$ ), Hoa Lai Style (from $10^{\text {th }}$ to $11^{\text {th }} \mathrm{c}$ ), My Son Style (from $10^{\text {th }}$ to $11^{\text {th }} \mathrm{c}$ ), Transition Style (from $11^{\text {th }}$ to $12^{\text {th }} \mathrm{c}$ ), Binh Dinh Style (from $12^{\text {th }}$ to $14^{\text {th }}$ c), Late Style (from $14^{\text {th }}$ to $17^{\text {th }}$ c) e with two different lay-out: central tower (one kalan), three aligned towers (three kalan).

The distribution of the archeological locations is visible in Figure 1. From $28^{\text {th }}$ of August to $4^{\text {th }}$ of September 2012 we visited and surveyed 19 archaeological sites. Near Hué, the ancient Vietnam capital, there is the most northern tower, Phu Dien, almost at sea coast line, (latitude $16^{\circ} 30^{\prime}$ longitude $107^{\circ} 40^{\prime}$ ) and the most southern tower is Pho Hai (latitude $10^{\circ}$ $56^{\prime}$ longitude $\left.108^{\circ} 09^{\prime}\right)$. The difference in latitude is then $5^{\circ}$ $44^{\prime}$. Vietnam has 54 ethnic groups, any of them having its own language and culture. For Champa group the most noticeable expression are the Champa towers, or Cham Temples. They are scattered within $1000 \mathrm{~km}$ all over the central and southern Vietnam. They are not as large as the Khmer temples in Cambodia, they were meant for worship only. The sanctum sanctorum has normally a Shivalinga in the centre. Thee Cham temples are normally square in plan with the main arched entrance, facing the east (the sunrise) and three blind doors on the other sides and decorated with carvings ad reliefs. The most used material for construction was brick; testifying the high standard in baking and assembling of bricks. They resisted in the rain forest for centuries. No binding material is visible between the bricks.

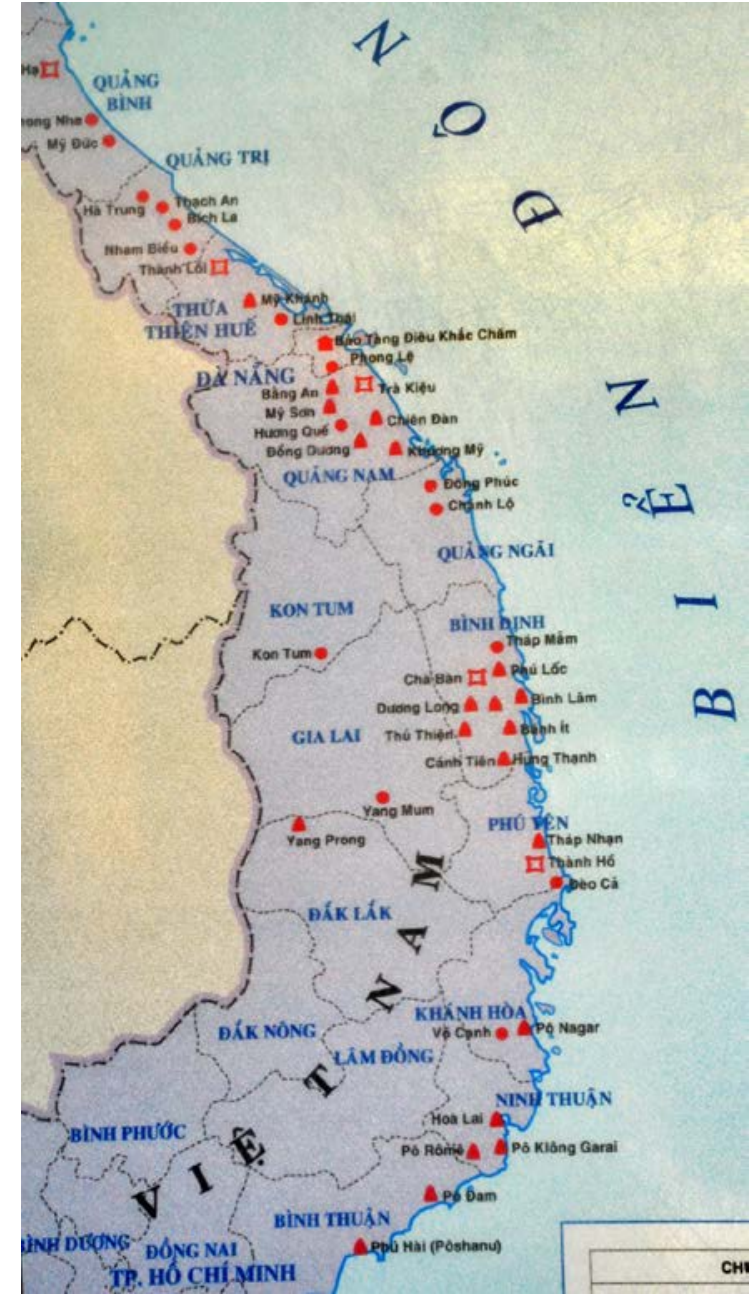

Figure 1. The location of the Cham Towers surveyed in Vietnam 


\section{THE PROJECT RUN WITH PSP}

Panoramic Spherical Photogrammetry (PSP) is a particular photogrammetric technique set up by the laboratory of the Engineering Faculty of Università Politecnica delle Marche (Ancona). It is based on the so-called spherical panorama, which is the cartographic representation of the sphere where are projected the images taken on the same point (Fangi, 2007, 2010, 2012).

Normally the survey is performed by making a traverse of panoramas around the monument, so that any point would be visible in at least three panoramas. In this project our equipment was only camera, tripod, spherical head and longimeter. The used cameras were a Canon Eos 1100D camera, 12 megapixels of resolution, equipped with 28, 50, $200 \mathrm{~mm}$ focal lenses, and a camera Canon B1X 14 megapixel with a zoom 15-55 mm. The images are stitched together and then mapped with the so-called longitude-latitude projection (Figure 2). The stitching software was PTGui 9. Normally FOV of a panorama was not $360^{\circ}$ but it was limited to the interested area only. The produced panorama is a quasihorizontal panorama. The orientation and restitution were run with Sphera package by (Fangi, 2012). Sphera package performs the model formation, the concatenation of the adjacent models up to the closing of the traverse, and finally the bundle block adjustment. The bundle block adjustment can be performed with six unknown parameters per pano. Only few known points are needed, (at least two, taken by a handheld Laser Distancemeter DISTO) and some extra geometrical conditions to overcome the rank deficiency of the system. From the image points the two directions of the straight line connecting the station point and the object point can be obtained, thus enabling the 3D model reconstruction, by intersection of two or more straight lines coming from different panoramas (Figure 3). The limit of the technique is essentially the lack of stereoscopy: only the well identifiable points can be plotted. In this very particular case the problem was the absence sometimes of sharp corners, the uniformity of materials, and of the colors, the erosion, not permitting in many cases the good identification of corresponding points. In table 1 some statistics of the project are shown, as far as PSP orientations.

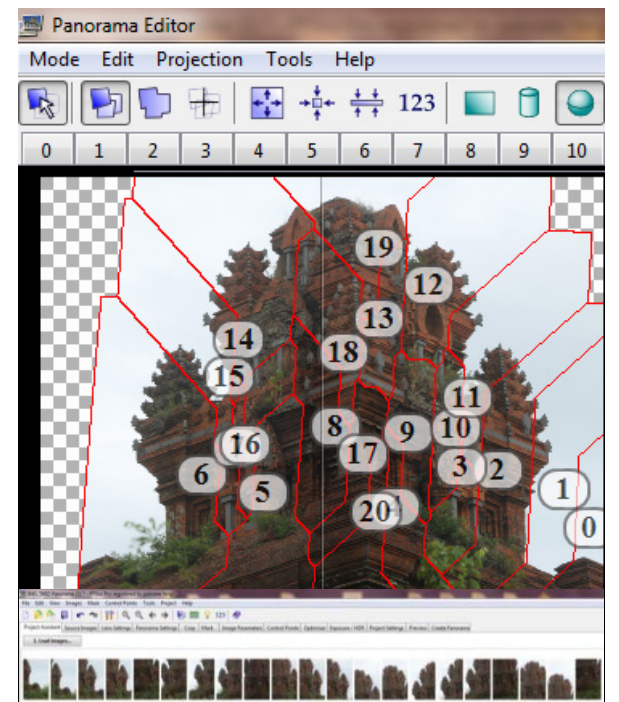

Figure 2. Image gallery and panorama editor (PTGui software)

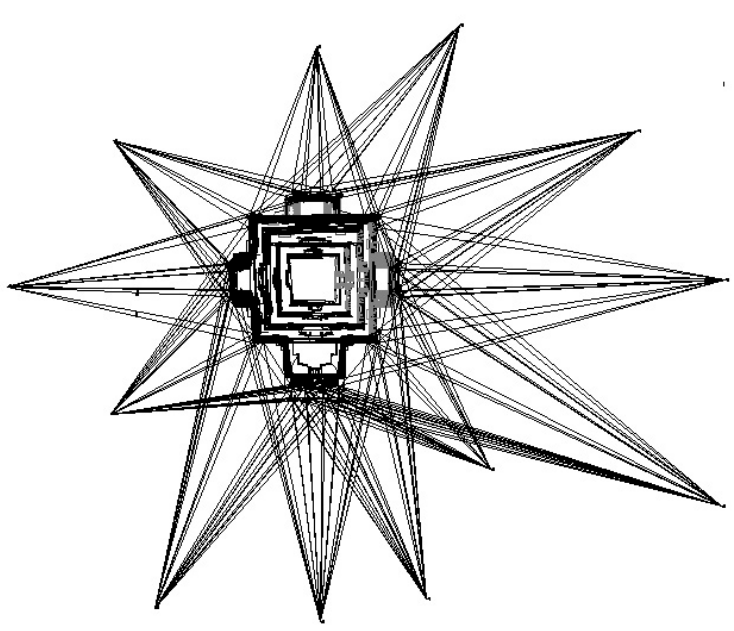

Figure 3. Network of oriented panorama (Hoa Lai tower)

Table 1 - Statistics of the PSP project orientations

\begin{tabular}{|l|l|l|}
\hline 14 PoNagar & 15 pano & Sigma-naught $=0.009 \mathrm{rad}$ \\
\hline 02 Bang An & 10 pano & Sigma_naught $=0.008 \mathrm{rad}$ \\
\hline 15 Hao Lai & 12 pano & Sigma_naught $=0.001 \mathrm{rad}$ \\
\hline 19 Pho Hai & 18 pano & Sigma_naught $=0.005 \mathrm{rad}$ \\
\hline 12 Hung Than & 06 pano & Sigma_naught $=0.007 \mathrm{rad}$ \\
\hline 13 Nahan & 13 pano & Sigma_naught $=0.0005 \mathrm{rad}$ \\
\hline 15 Hoa Lai & 13 pano & Sigma_naught $=0.002 \mathrm{rad}$ \\
\hline
\end{tabular}

Sigma_naught of the adjustments varies from 9 to 0.5 thousandths of radiant, this means that the accuracy ranges from $1 / 100$ to $1 / 1000$ of the object distance. This has not been very high, but you have to remember that the aim of the project was the archaeological site documentation only.

\section{PSP RESTITUTION FOR CHAM TOWERS DOCUMENTATION}

The orientation stage by PSP produced a set of oriented panorama useful for 3D model outputs. The first product was line plotting tridimensional wireframe model. The model was then textured and rendered making use of the original rectified image. We illustrate the results of to the most meaningful examples of Cham heritage (Nguyen Van $\mathrm{Ku}$, 2008), limited to the wireframe models only.

\subsection{The Bahn It site}

The name of the complex comes from the shape of the tallest tower with its peak which looks like a Banh It (threecornered glutinous rice cake filled with green bean paste and meat). Each tower has been built with different design and architecture and varied shape, creating diversity in the complex.

The main tower is 22 meters high and the architecture bears the Binh Dinh sculptural style of the 12th century with small, embossed lines on the walls (Figure 4). Leaf and flowershaped motifs are only found on the edges of the roofs, and dancing girls are found on false gates. 


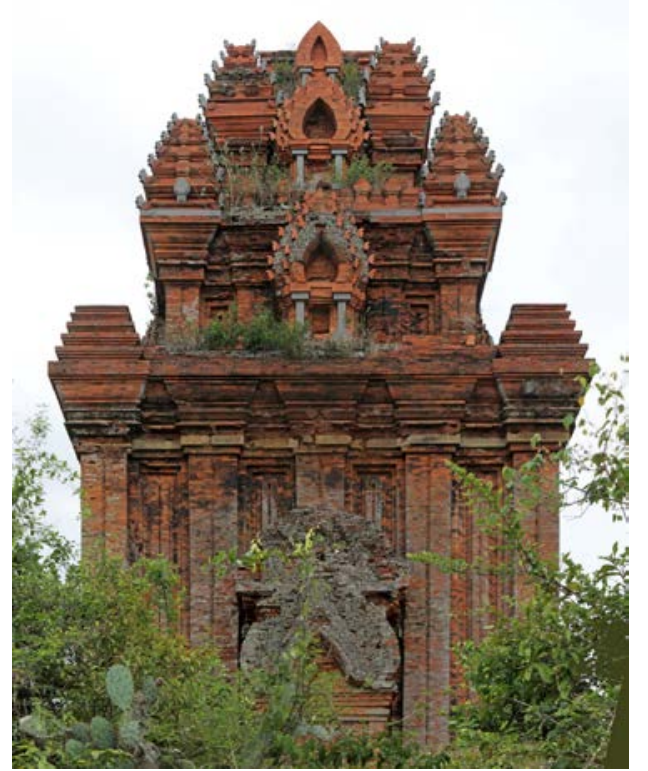

Figure 4. Banh it tower. Its peak looks like a Banh It (threecornered glutinous rice cake filled with green bean paste and meat).

\subsection{The Hoa Lai site}

Hoa Lai Tower group was built from IX century. Originally, the group had 3 towers: East Tower (Figure 5, 6), Central Tower and West Tower (Figure 7). The Central Tower was uncompleted so that it is now left its foundation only.

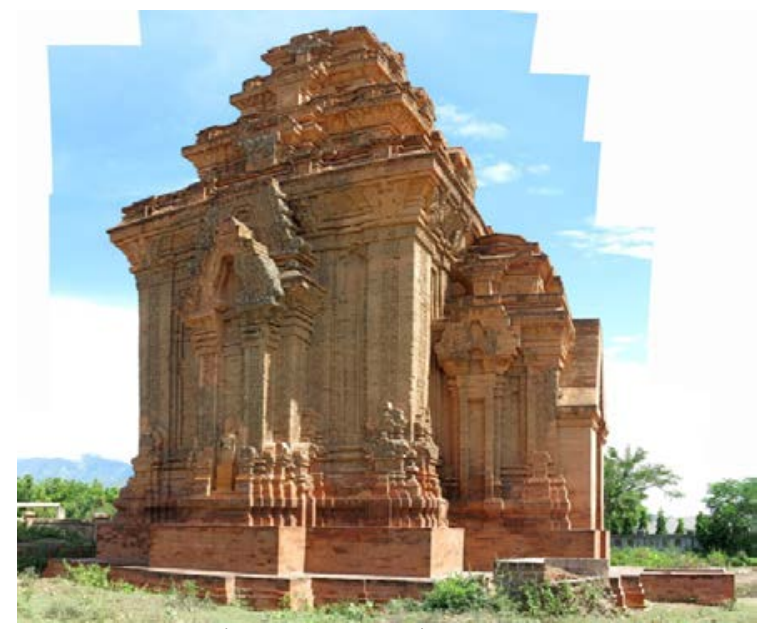

Figure 5. Hoa Lai East tower

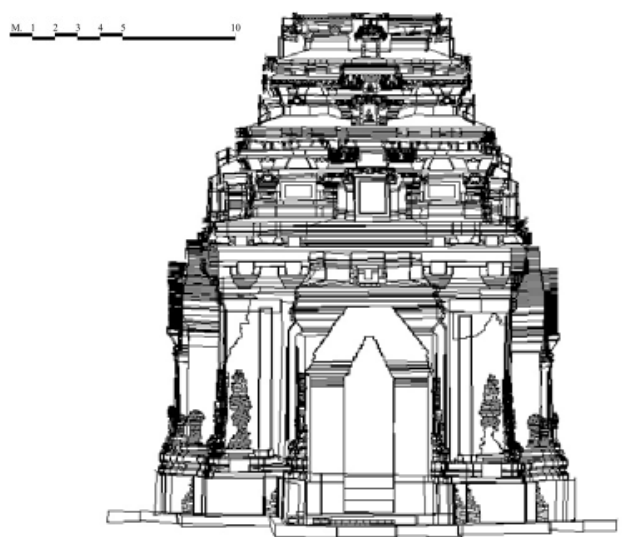

Figure 6. Hoa Lai East tower line plotting
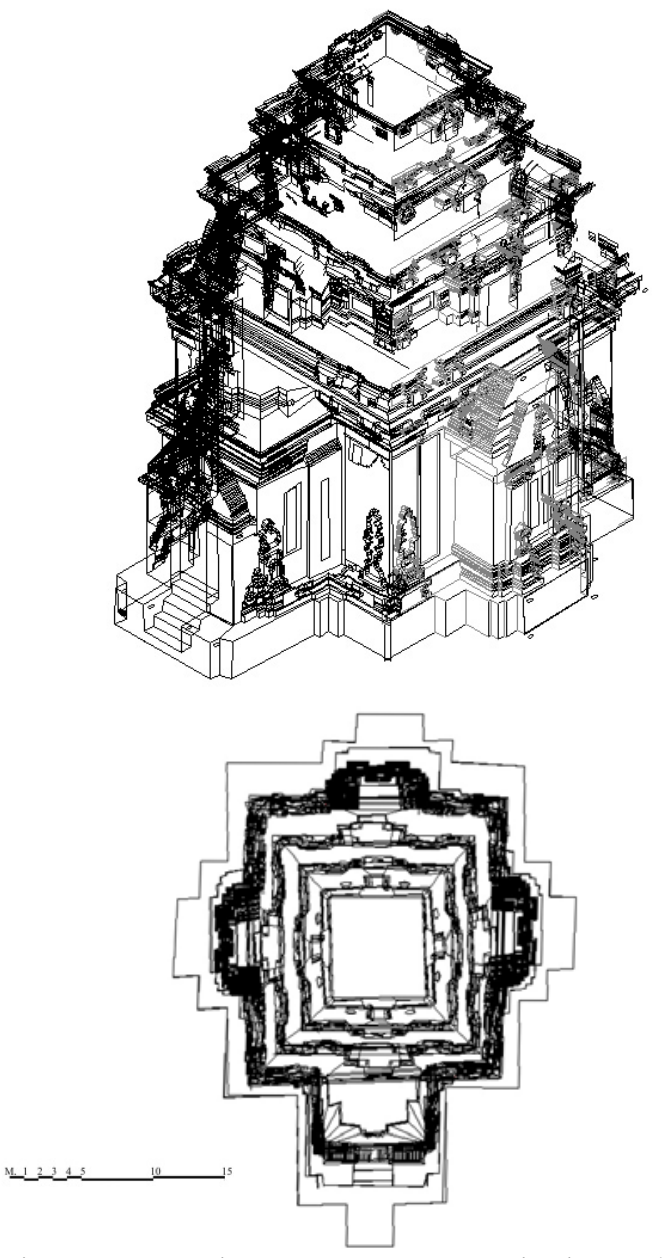

Figure 7. Hoa-Lai West tower axonometric view and plan

Hoa Lai Tower group is very valuable in architecture and sculpture of Cham people. Therefore, Hoa Lai was said to open a new style of Cham architecture Hoa Lai style.

The East Tower was high and built with bricks. The West Tower was higher and also built with bricks and its brick walls were skillfully sculptured patterns but it is now in draft only.

\subsection{The Po Klong Garai site}

The temple of Po Klong Garai belongs to what is known as the Thap Mam Style of Cham art and architecture. It consists of three brick towers: The group is well preserved (Figures 8, $9,10)$.

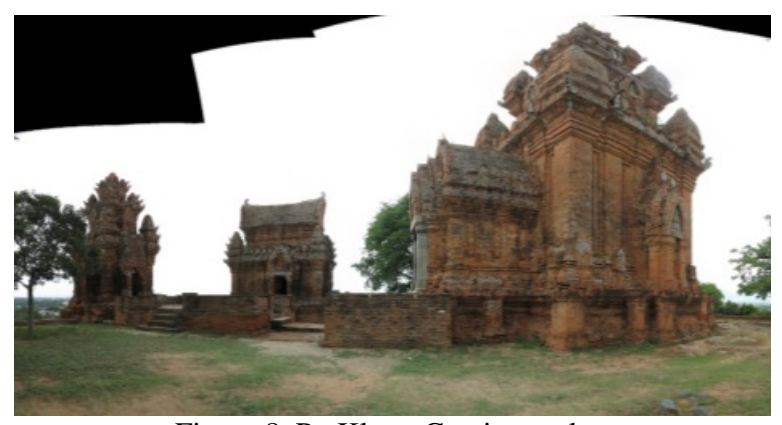

Figure 8. Po Klong Garai complex

The central tower was built in honor of the legendary king Po Klong Garai by the historic King Jaya Simhavarman III, who ruled Champa from 1285 to 1307. 

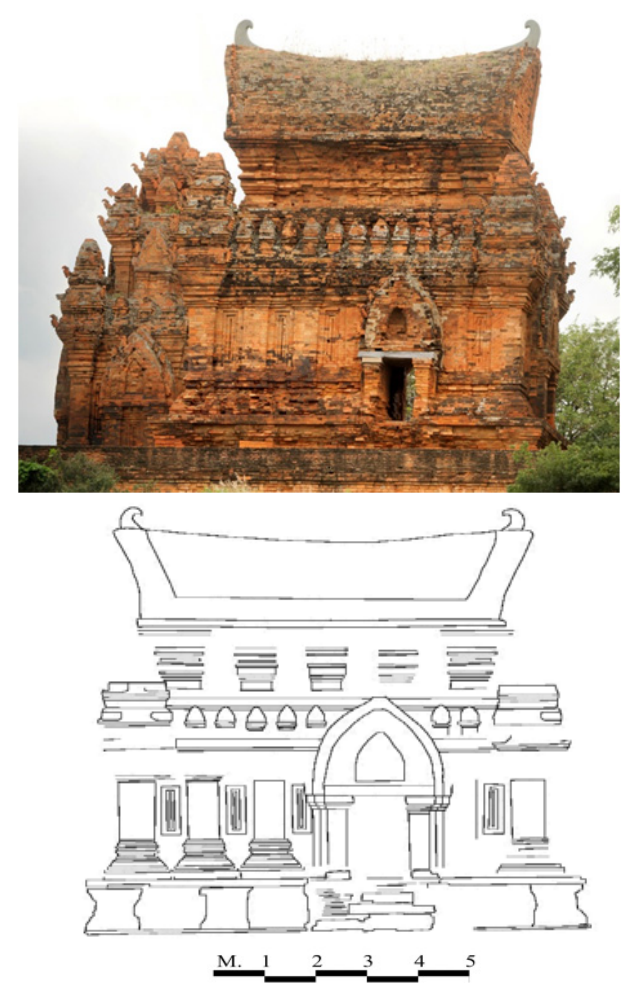

Figure 9. Po Klong Garai tower and line restitution. The long-saddle shaper curved roof is its characteristic

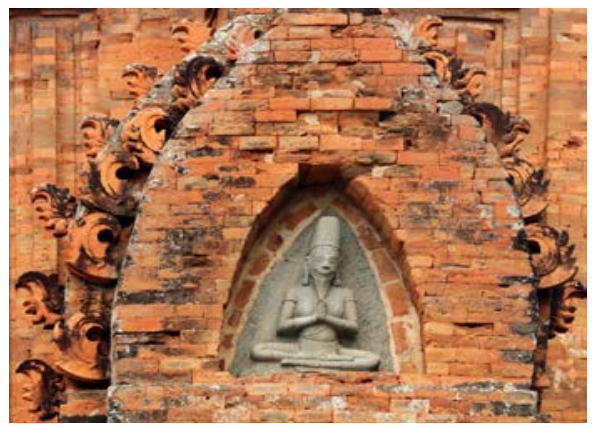

Figure 10. The sculpture of the God Shiva regarded as one of the masterpieces of the Thap Mam Style

\subsection{The Po Nagar site}

Po Nagar is a Cham temple site founded before 781 (Figure 11, 12). The Po Nagar complex is situated on Cù Lao Mountain. It consists of three levels, the highest of which encompasses two rows of towers. The main tower is about $25 \mathrm{~m}$ high.

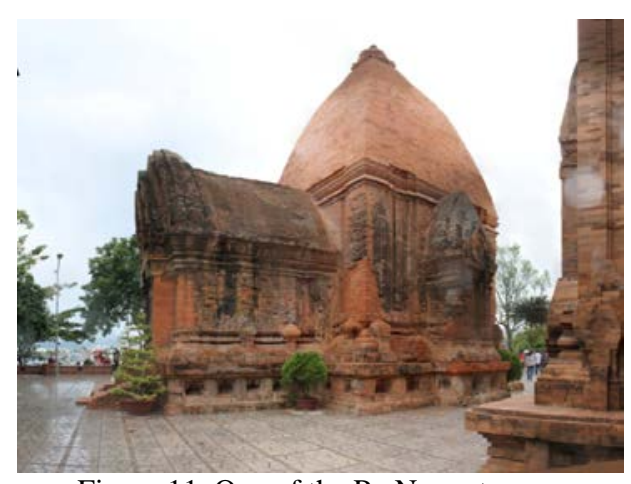

Figure 11. One of the Po Nagar towers

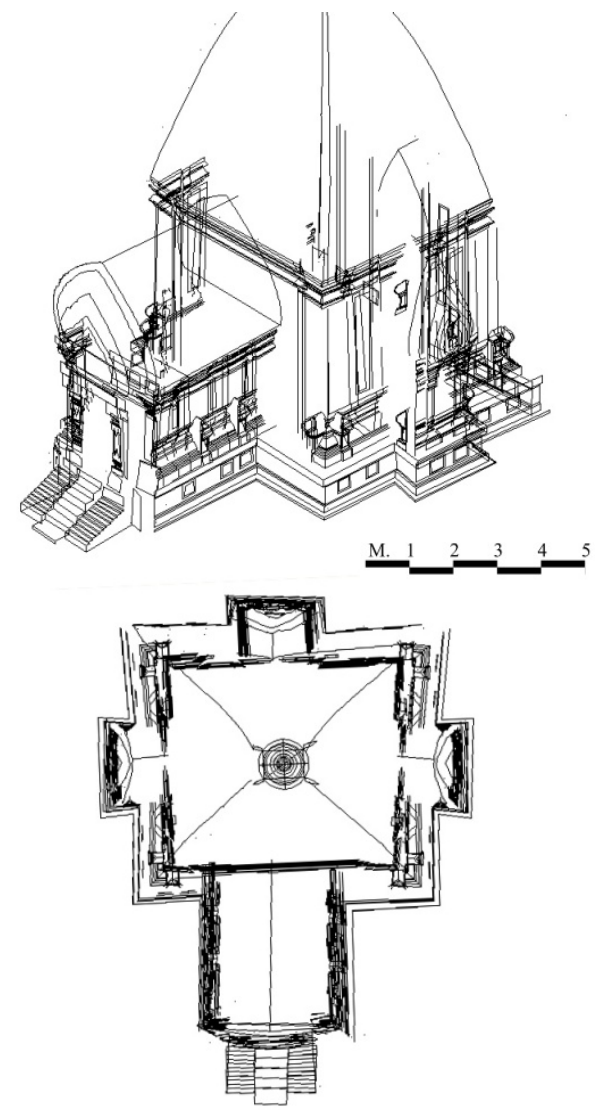

Figure 12. Po Nagar axonometric view and plan

\section{POINT CLOUDS BY LASER SCANNING AND SfM}

In 2010 a small sample test of four towers only has been surveyed by laser scanner. The point cloud was acquired with the laser Z+F IMAGER 5006h and processed by means of Cyclone software (Figure 13).

An example of this type of surveying is related to the Thu Thien tower. It is a square tower of $12^{\text {th }}$ century with three storeys, $20 \mathrm{~m}$ high. It has three false doors and miniatures towers at the corners.

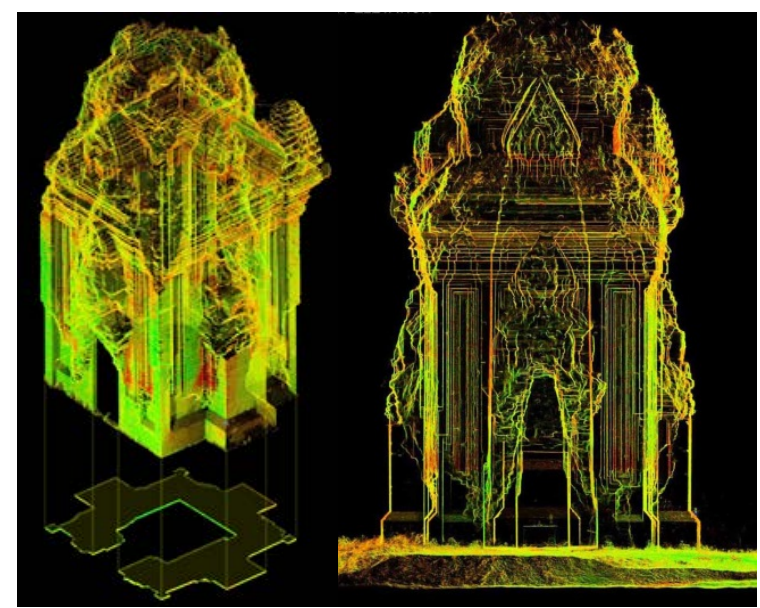

Figure 13. Thu Thien tower. One or the four towers surveyed by laser scanner

The complex of three towers of Khuong My is important for its stone sculptures and for the carved figures which are a passage from the traditional Cham art to a new style called just Khuong My Style ( $10^{\text {th }}$ century). The walls are another 
example of laser scanning surveying. The range maps were aligned and used for the line plotting restitution of the front and the plan (Figure 14).
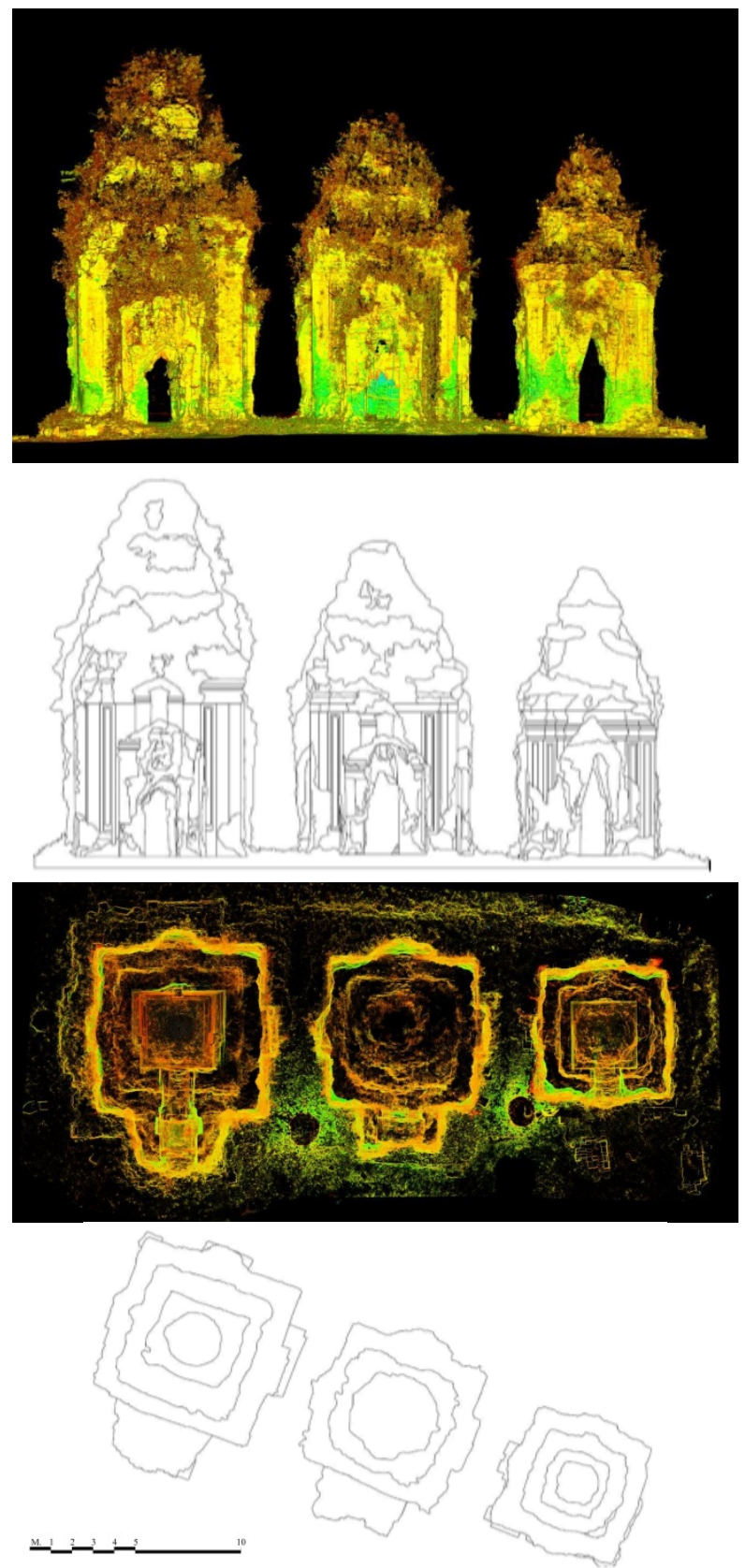

Figure 14. The Khuong My survey and graphical outputs

Another technique applied to produce point clouds has been based on digital photogrammetry by means of Structure from Motion (SfM) algorithm applied by Agisoft Photoscan (http://www.agisoft.ru/products/photoscan). The software is an advanced image-based 3D modeling solution based on the latest multi-view 3D reconstruction technology. It operates on arbitrary images that can be taken from any positions, with the only constrain that the object was visible on at least two photos. Both image alignment and 3D model reconstruction is fully automated (Doneus et al., 2011).

In Figure 15 one of the three Po Dam Towers processed by means of a selection of 37 overlapped panorama images. The software has given a dense mesh and a textured 3D model. Orientation and scale can be provided. The mesh is made of more than 3,5 millions of triangles (Figure 16).
Po Dam is one of the oldest complexes; it was built in early $9^{\text {th }}$ century. It consists in 6 towers, divided in two groups: 3 small front towers and behind only one existent respect the three original.

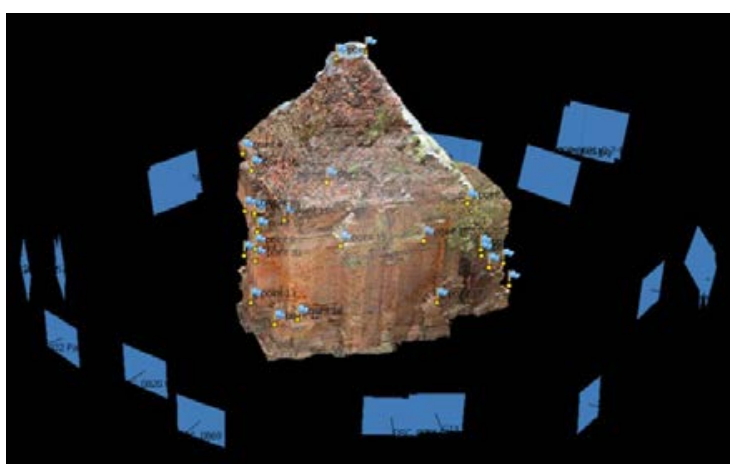

Figure 15. Po Dam Tower, the achieved 3D textured model by Photoscan Agisoft (SfM)

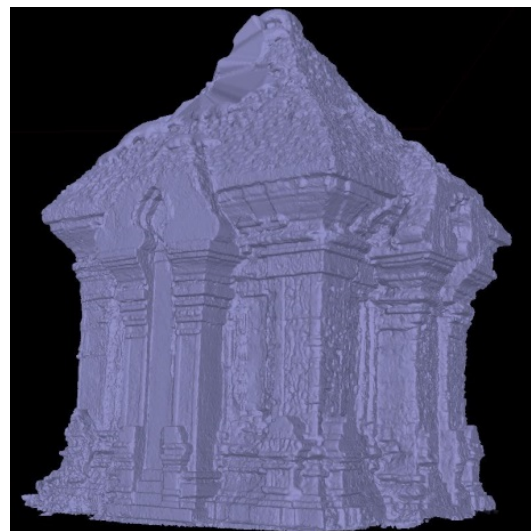

Figure 16. Po Dam Tower, the achieved 3D mesh by Photoscan Agisoft (SfM)

\section{GIS DOCUMENTATION AND CHAM HERITAGE VIRTUAL TOUR}

The documentation of archaeological sites and monuments plays an essential role in promoting the understanding, conservation and preservation of the archaeological heritage. When the architectures are diffused in a large territory and relatively accessible, in so large amount, some tools are needed to help to collect and manage in organized form the data for the experts or to introduce them to the community. The cataloguing in standard format of every available data allows deciding the priorities and the type of actions for the maintenance and the restoration of the monuments. We give an example of a dedicated GIS, collecting and organizing the information about the surveyed Towers: localization, name, date, style, material, images which can be managed in a database. But many others information can be added putting in relationships with the environmental and human elements, and with the other heritage goods close to it, offering the best and the most aware knowledge of themselves. These particular requirements are satisfied by means of GIS.

Moreover starting from the data acquired during the project we realized some links in the GIS to summarize the best knowledge of the Cham Heritage by "descriptive" card (Figure 17) or by virtual tour to explore the sites (Figure 18). 


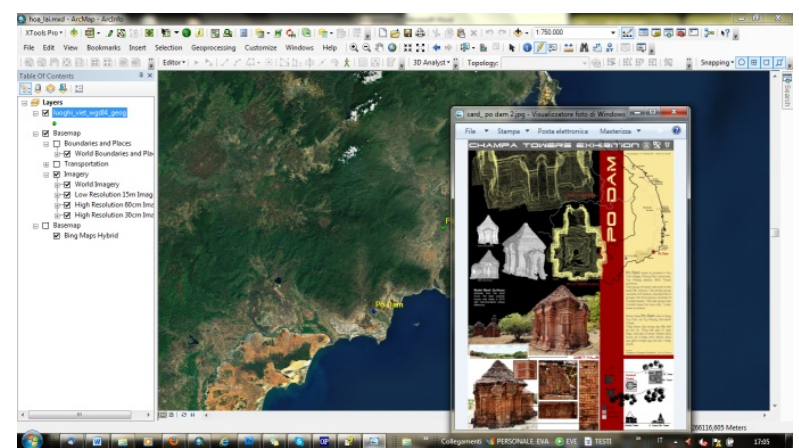

Figure 17. A description of the site by a hyperlink card in the GIS database

A "virtual tour" or a "panoramic tour" is a simulation of an existing location, usually composed of a sequence of images, sometimes adding other multimedia elements such as sound effects, music, narration, and text. The starting point necessary to create a virtual tour is fundamentally the $360^{\circ}$ panorama. The first use of a virtual tour and the derivation of the name was in 1994 as a museum visitor interpretation, providing a 'walk-through' of a 3D reconstruction of Dudley Castle in England as it was in 1550. Since this date its applications increase more and more. The methods of creation is based on the image stitching by means of one of the popular ways: equirectangular stitching. This involves the rotation of a digital camera, centered directly over the tripod. The combination of a precision rotator and a digital camera allows the photographer to take rectangular "slices" of any scene (indoors or outdoors). With a typical point and shoot digital camera, the photographer will snap $8,10,12$ or 14 slices of a scene. Using specialized "photo stitching" software such as PTGui and Pano2VR (GardenGnome) to manage the panorama, one can create media rich virtual tours. Virtual tours are used extensively for universities and this can allow a user to view an environment whilst on-line. Over the last few years the quality, usability and accessibility of virtual tours has improved considerably, with some websites allowing the user to navigate the tours by clicking on maps. For most business purposes, a virtual tour must be accessible from everywhere. The major solution is a web-based virtual tour. Thanks in part to the recent explosion of many Internet devices, such as Apple's iPad, iPhone and other tablet computing platforms, it can be predicted that consumption of virtual tour content, through the use of HTML5/CSS3 driven virtual tours will only increase over time.

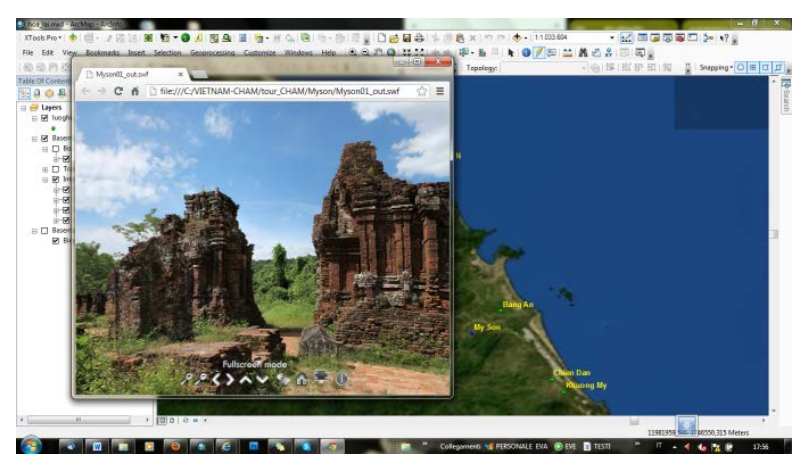

Figure 18 Virtual tour joint in the GIS database

\section{CONCLUSIONS}

We used three different methods of survey, laser scanning, point clouds formation by SfM software, and finally PSP or Panoramic Spherical Photogrammetry. The most used was PSP, for the simplicity and economy of the instrumentation, its lightness and easiness. It is indubitable that the ideal instrument for this complex architecture is the laser scanning. But its availability in the area is very limited for practical and economical reasons. In any case PSP demonstrated its validity for the speed of acquisition, and the completeness. We have no information on the accuracy of the survey except through the intrinsic block adjustment output. On the other end Agisoft Photoscan was used to test its validity and the possibility to integrate it inside the PSH procedure. In the restitution phase the intelligent selection of the information with PSP produces good guided restitution. PSP is characterized by speed of execution in the taking phase; at the moment it is still time consuming in the plotting phase. But if we want to build a data base of the monuments, there is no need to finish now the plotting and produce drawings. This final phase can be performed at any time, according to the needs. We do not have information about the accuracy of the survey, because we didn't perform any dedicated task, and after all this was not the task of the mission. The main problem for Spherical Photogrammetry is after all the lack of stereoscopy and the lack of any automation in the orientation and restitution phases. The Champa towers project is up to now the largest project run with the spherical photogrammetry.

\section{Aknowlodgements}

E. Alimento, R. Angeloni, M. Bigoni, C. A. Bozzi, M. Brunetti, A. Caporossi, G. Caruso, N. Cataldo, S. Ferini, V. Friuli, B. Lozzi, L. Lupini, C. Pascucci, A. Piccioni, S. Pierantozzi, L. Piergiovanni, L. Piermattei, E. Raffaelli, F. Silvestri, G. Issini produced the restitution of the towers during the course of Photogrammetry. Thanks to all of them.

\section{REFERENCES}

Doneus, M., Verhoeven, G., Fera, M., Briese, Ch., Kucera, M., Neubauer, W., 2011. From deposit to point cloud - a study of low-cost computer vision approaches for the straightforward documentation of archaeological excavations. In: Geoinformatics, CTU FCE 2011, pp. 81-88.

Guidi, G., Remondino, F., Russo, M., Menna, F., Rizzi, A., Ercoli, S, 2009. A multi-resolution methodology for the 3D modeling of large and complex archaeological areas. International Journal of Architectural Computing, 7 (1), pp. 39-55.

Fangi, G., 2007. The Multi-image spherical Panoramas as a tool for Architectural Survey. In: ISPRS International Archives, Vol. XXXVI, Part 5/C53, CIPA Archives, Vol. XXI, pp. 311-316.

Fangi, G., 2010. Multiscale Multiresolution Spherical Photogrammetry With Long Focal Lenses For Architectural Surveys. In: ISPRS International Archives, Vol. XXXVIII, Part 5, pp. 228-233.

Fangi, G., Pierdicca, R., 2012. Notre Dame du Haut by Spherical Photogrammetry integrated by Point Clouds generated by Multi-view Software. International Journal of Cultural Heritage in the Digital Era, Vol. I, n.3, pp. 461-478.

Nguyen, Van Ku, 2008. Cham Heritage of Cham Culture. The Gioi Publishers, Hanoi (Vietnam), 2008.

Pisa, C., Zeppa, F., Fangi, G., 2010. Spherical Photogrammetry for Cultural Heritage - San Galgano Abbey, Siena, Italy And Roman Theatre, Sabratha, Libya. In: ACM Journal on Computing and Cultural Heritage, Vol. 4, No. 3 